\title{
Nanoindentation on Graphene Encapsulated Single Cells
}

\author{
Jiayao Li ${ }^{1}$, Changxi Zheng ${ }^{2}$, Boyin Liu ${ }^{1}$, Yeonuk Kim${ }^{1}, \mathrm{Jian} \mathrm{Li}^{3}$, Wenyi Yan ${ }^{1}, \mathrm{Jing} \mathrm{Fu}^{1}$
}

${ }^{1 .}$ Department of Mechanical Engineering, Monash University, Clayton, VIC, Australia.

2. Department of Civil Engineering, Monash University, Clayton, VIC, Australia.

${ }^{3 .}$ Monash Biomedicine Discovery Institute, Department of Microbiology, Monash University, Clayton, VIC, Australia.

Investigations on liquid-phase samples with high-resolution imaging and probing attract increasing attentions in recent years. The rapidly developed nanotechnologies enable researchers to dynamically monitor the interior changes occurred within living single cells [1]. Silicon membrane technology, as a popular strategy for fabricated window in electron microscopy, has been used for imaging hydrated specimens. However, there is a trade-off between image resolution and the robustness of windows, and the fabricated windows tend to deform in different modes during applications approaching few-nanometre resolution [1]. Furthermore, the over-thickened Si film over the liquid sample is not applicable for additional scanning probe microscopy. In this study, an alternative approach that utilizes graphene to encapsulate hydrated samples such as biological cells, is proposed due to its extraordinary physical properties, and a computational framework to explore the fundamental mechanics is presented.

Monolayer graphene film was grown based on the chemical vapour deposition (CVD) approach. A drop of solution containing bacterial cells was injected between the floating graphene film and the glass substrate. Dehydration of the whole sample results in adhesion of the graphene film with the hydrated cells encapsulated, and Atomic Force Microscopy (AFM) could be performed to acquire the topological information (Fig. 1a). In addition, force mapping experiments could be implemented, and Finite Element Analysis (FEA) was also established in parallel to model the dynamics of the AFM tip with the multilayered structure (Fig. 1b). Parameters such as the mechanical properties were sourced from either literature or data from manufacturer, with the exception of modulus of targeted hydrated sample. All the involved surface-surface interactions were assumed to be frictionless [2]. The established model without graphene was verified by applying the Hertz contact theory, whereas the model with graphene was experimentally verified by introducing a reference/calibration sample of Polydimethylsiloxane (PDMS) with determined modulus. With the verified models, the simulated force-displacement (FD) curves were reversely fitted to determine the modulus of the hydrated sample underneath.

Results reveal that FD curves are significantly affected with the addition of a graphene layer, with enhancement on modulus estimated by the simulation as shown in Fig. 1c. The results also imply that a stiffer sample wrapped by graphene leads to a relatively minor enhancement on modulus. This proposed approach offers a novel and simplified solution for acquiring force maps of liquid/live cells without additional liquid environment accessories [3] that are essentially required in the conventional AFM approaches. As a demonstration, the modulus acquired on a hydrated bacterial cell (Klebsiella pneumonia) is assessed to be approximately $300 \mathrm{kPa}$, by fitting the experimental data to the FD curves sourced from FEA results (Fig. 1d). In summary, the successful establishment of experimental and modelling framework of graphene encapsulation provides a new approach for probing liquid/live cells with scanning probe microscopy and potential for correlative imaging of hydrated samples [4]. 


\section{References:}

[1] de Jonge N, Ross FM. Nature Nanotechnology 6 (2011), p. 695.

[2] Raman A, et al. Nature Nanotechnology 6 (2011), p. 809.

[3] Li Q, et al. Biochemical and Biophysical Research Communications 374 (2008), p. 609.

[4] Acknowledgment: This study was funded by Monash University Interdisciplinary Research (IDR) Seed Fund. Jiayao Li is supported by Australian Government Research Training Program scholarship and PhD top-up scholarship from Monash Centre for Atomically Thin Materials (MCATM). This work was performed in part at the Melbourne Centre for Nanofabrication (MCN), Victorian Node of the Australian National Fabrication Facility (ANFF). Also, the authors acknowledge use of facilities within the Monash Centre for Electron Microscopy (MCEM).

a
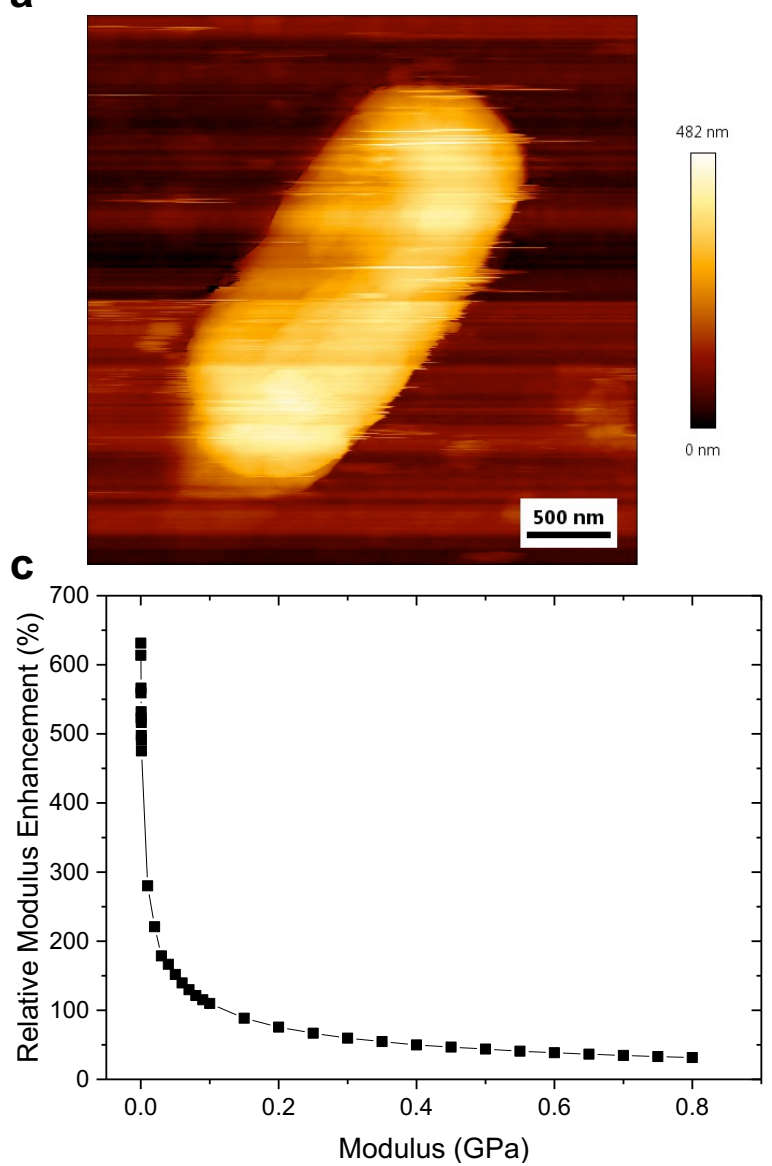

b

d
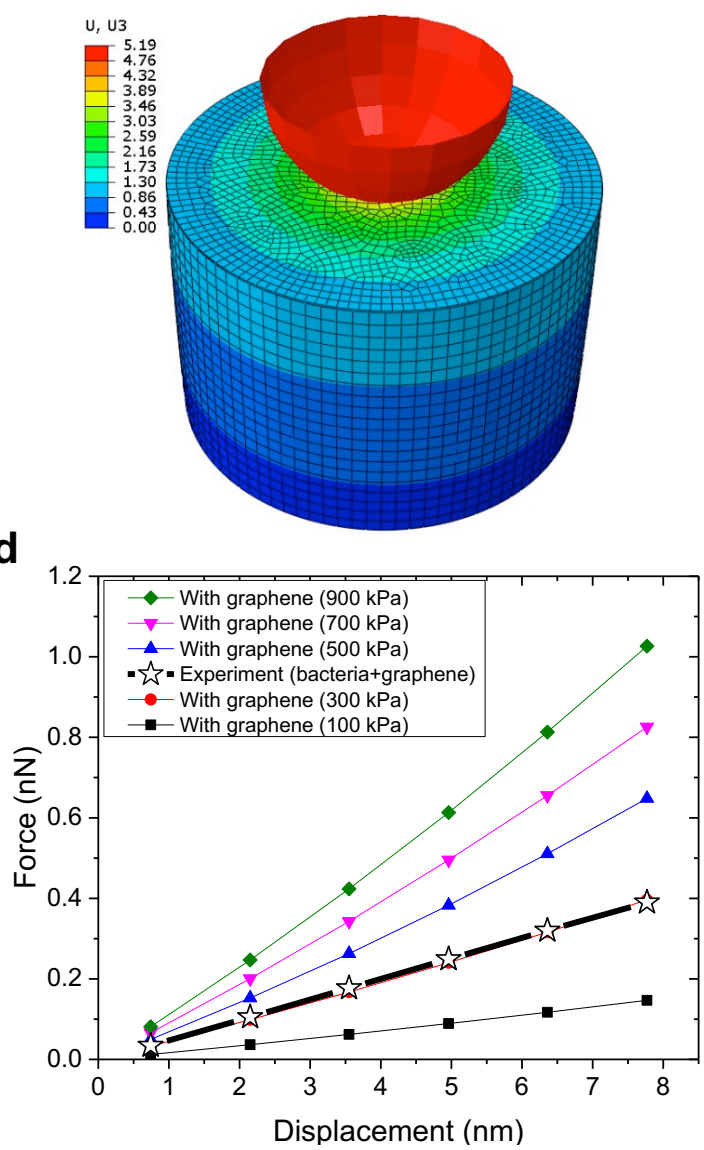

Figure 1. (a) Topographic AFM image of a graphene-encapsulated bacterial cell; (b) The constructed FEA model of the AFM tip, graphene layer and the target sample; (c) Relative modulus enhancement measured with AFM force spectroscopy with the addition of graphene layer on targeted samples based on original modulus; (d) Simulated force-displacement curves of indentation on graphene encapsulated materials of modulus range in $100-900 \mathrm{kPa}$, with experimental data curve of bacterial cell fitted at 300 $\mathrm{kPa}$. 